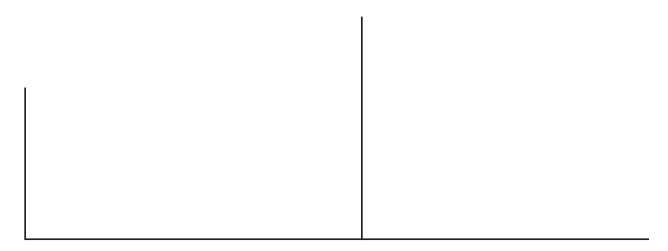

Rev. Latinoam. Psicopat. Fund., II, 2, 52-71

\title{
Transtorno borderline: história e atualidade
}

\author{
Paulo Dalgalarrondo e Wolgrand Alves Vilela
}

No presente trabalho é feita uma revisão histórica e psicopatológica do distúrbio borderline. O autor sugere que a categoria clínica introduzida por Kahlbaum, em 1890, "heiboidophenie", pode ser considerada como um protoconceito na origem do conceito de transtorno borderline. No final dos anos 70 e 80, sob a influência do DMS-III, a esquizofrenia latente ou borderline se transformava em dois subtipos de transtorno de personalidade: borderline $e$ esquizotípico. Alguns aspectos psicopatológicos do Transtorno de Personalidade Borderline são revistos, com especial ênfase sobre os sintomas afetivos e as alterações no plano das relações interpessoais.

Palavras-chave: Transtorno de personalidade borderline, esquizofrenia latente, história da psicopatologia. 


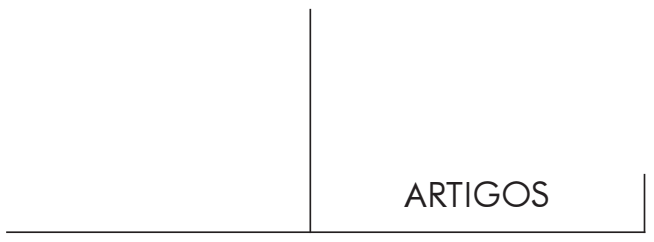

Introdução

O transtorno borderline surge como categoria diagnóstica utilizada de modo mais amplo na clínica psiquiátrica e psicanalítica no princípio da década de 50 (Masterson, 1972) ${ }^{1}$. A noção de borderline constitui-se inicialmente como uma entidade vaga e imprecisa, que compreende sintomas que se estendem desde o espectro "neurótico", passando pelos "distúrbios de personalidade", até o espectro "psicótico".

Desde então, o quadro tem sido freqüentemente diagnosticado em adolescentes e adultos jovens com comportamento impulsivo e/ou autodestrutivo, uso de drogas e com problemas sérios de identidade, notando-se um predomínio no gênero feminino (por volta de $75 \%$ dos casos) (APA, 1994). Esses indivíduos mal se encaixam entre as neuroses graves ou entre as psicoses endógenas clássicas. Ao longo das últimas duas décadas o conceito vem ganhando popularidade e também uma maior precisão. Além disso, a síndrome borderline tem sido estudada de forma crescente por clínicos e pesquisadores; um levantamento no sistema medline de catalogação computadorizada de artigos médicos, revela, nos últimos 10 anos, mais de 1.500 artigos publicados sobre o tema "transtorno de personalidade borderline".

$\mathrm{O}$ conceito atual de borderline foi aquele formulado inicialmente para a classificação norte-americana das doenças mentais de 1980 , o DMS-III ${ }^{2}$. Neste sistema diagnóstico, a síndrome borderline deixa de ser uma acepção relativamente vaga de estados intermediários neurose-psicose, para ser um distúrbio específico de personalidade, no qual comportamentos impulsivos, autolesivos, sentimentos de vazio interno e defesas egóicas muito primitivas seriam predominantes.

Este trabalho tem fundamentalmente dois objetivos. De um lado, descrever em linhas gerais a evolução do conceito de borderline e ressaltar a necessidade de uma definição mais precisa desta entidade; de outro, apresentar o quadro psicopatológico e alguns mecanismos mentais propostos para esses pacientes.

\section{Evolução histórica do conceito de borderline}

A necessidade de se criar categorias diagnósticas para distúrbios mentais graves, próximos da alienação mental, mas sem sintomas declarados de "loucura",

1. J.F. Masterson. "Psicoterapia intensiva del adolescente con un Síndrome Borderline", in El adolescente borderline. Cuademos de la ASAPPIA. Buenos Aires, Kargieman, 1972.

2. DSM-III (APA) (1980). Diagnostic and Statistical Manual of Mental Disorders, $3^{\text {rd }}$ ed.. Washington, DC, American Psychiatry Association, 1980. 


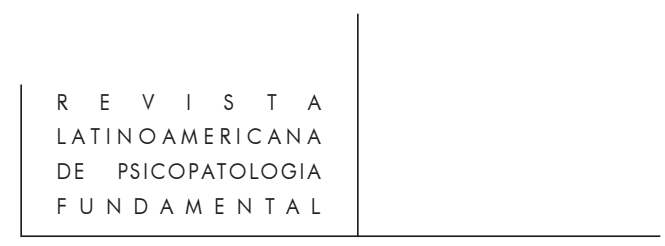

não é nova na tradição psicopatológica (Berrios, 1993) ${ }^{3}$. Nesse sentido, entidades como a moral insanity de Prichard (1835), a manie sans délire de Pinel, e as monomanias de Esquirol (1838), poderiam ser tomadas como precursores do conceito de borderline. Para o alienista Chaslin, haveria de fato um território fronteiriço, entre a loucura e a sanidade, uma borderland of insanity, como ele sugeriu na virada do século, digna de ser estudada com maior cuidado pelos alienistas.

É, entretanto, apenas no final do século passado que uma categoria bastante semelhante ao que hoje se configura como transtorno borderline passou a se delinear mais claramente. Assim, Kahlbaum $(1884,1890)^{4,5}$, criador do conceito de catatonia, e junto com seu discípulo Hecker, do conceito de hebefrenia, descrevem com detalhes uma síndrome comum a adolescentes, que, apesar de aparentada com a hebefrenia, se assemelharia ao que hoje é concebido como transtorno borderline.

Em 1871, Ewald Hecker ${ }^{6}$ descreve de modo detalhado e brilhante (Sadler, $1985)^{7}$ uma forma de loucura própria de adolescentes, que se caracterizaria por um afeto "bobo", "pueril", por alterações comportamentais graves e desagregação progressiva do pensamento. Seria o que Kahlbaum? denominara de "vesânia ou loucura juvenil propriamente dita". Em um trabalho de 1884, sobre as doenças mentais dos jovens, Kahlbaum fala de quadros menos graves, comuns a adolescentes, 54 nos quais predominam alterações do comportamento ético e social. É, entretanto, no trabalho intitulado "Sobre a heboidofrenia", de 1890, que Kahlbaum ${ }^{8}$ irá descrever com detalhes essa forma frusta e curiosa de alienação juvenil.

$\mathrm{Na}$ heboidofrenia as alterações seriam principalmente na esfera das relações sociais e de personalidade. Surgiriam nesses adolescentes alterações na esfera instintiva, distúrbios da compreensão das regras morais e dos hábitos culturais. Nos casos extremos poderiam surgir também tendências ou evidentes comportamentos delinqüentes. Para Kahlbaum haveria dois tipos básicos de transtornos mentais específicos dos adolescentes que ele delimitou no grupo dos transtornos hebéticos. Em primeiro lugar a hebefrenia, com sintomas evidentes de loucura e, em segundo,

3. G.E. Berrios. "European views on personality disorders: a conceptual history". Compr. Psychiatry, 34 (1): 14-30, 1993.

4. K. Kahlbaum. "Ueber jugendliche Nerven - und Gemuethskranke und ihre paedagogische Behandlung in der Heilanstalt”. Allgemaine Zeitschrift fuer Psyshiatrie, 40: 863-873, 1884.

5. K. Kahlbaum. "Ueber Heboidophrenie". Allgemaine Zeitshrift fuer Psychatrie, 46: 461-474, 1890.

6. E. Hecker. "Die Heboidophrenie". Achiv fuer pathologische Antomie und psysiologische und fuer klinische Medizin, 25: 394-429, 1971.

7. K. Kahlbaum. "Ueber jugendliche Nerven...". Op. cit.

8. K. Kahlbaum. "Ueber Heboidophrenie”. Op. cit. 


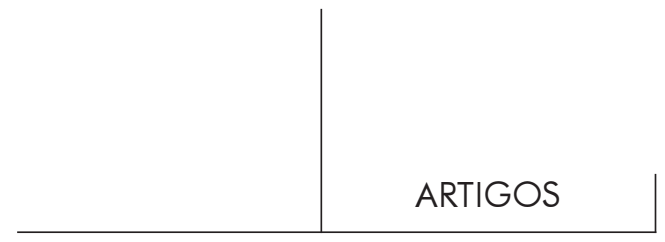

a heboidofrenia, aparentada com a hebefrenia, mas diferindo dela por não evidenciar sintomas claros de alienação. A heboidofrenia seria menos desastrosa que a hebefrenia; o doente não perderia as capacidades cognitivas básicas e o quadro não evoluiriam para estados terminais confusionais ou deficitários. O adolescente hebóide poderia se apresentar aos olhos menos atentos simplesmente como um jovem maleducado, com tendências a flutuações abruptas do humor e do comportamento. Oscilam rapidamente de um estado melancólico para um expansivo, ficando facilmente irados. São jovens que, embora pensem e raciocinem corretamente, discutindo os mais variados assuntos com facilidade têm, de fato, uma certa dificuldade em apreender de forma precisa a realidade. Entretanto, apesar desta forma de pensar dispersa e pouco útil, os hebóides, ao contrário dos hebefrênicos, não apresentam idéias delirantes verdadeiras.

No começo do século, os trabalhos de Eugen Bleuler ${ }^{9}$ sobre a esquizofrenia vão trazer novas contribuições para este tema. Na sua monumental monografia de 1911, "Dementia praecox, ou o grupo das esquizofrenias", o psiquiatra suíço relata a existência de um grupo de pacientes que, apesar de apresentarem um comportamento social convencional, trariam consigo, de modo subjacente, elementos de esquizofrenia. Este quadro ele denomina, "esquizofrenia latente", reconhecendo, entretanto, sua dívida para com a heboidofrenia de Kahlbaum. Bleuler (1911) descreve esse tipo de paciente da seguinte forma ${ }^{10}$ :

Kahlbaum cunhou o termo "heboidofrenia" ou "hebóide" para aqueles casos que mostram apenas transtorno dos sentimentos sociais, do tato social e do comportamento. (...) entretanto, os casos que ele descreveu nesse grupo tomam um curso que não difere de outros casos leves de esquizofrenia.

(...) outro tipo, com uma pronunciada irritabilidade: uma jovem normal e inteligente se casa aos vinte anos e vive feliz por mais de cinco anos. Muito gradualmente torna-se irritável, gesticula enquanto fala, suas peculiaridades aumentam continuamente; contudo, nenhuma empregada pára em casa. Briga constantemente com seus vizinhos. Dentro de seu próprio grupo familiar converteu-se numa tirana doméstica insuportável que não reconhece seus deveres, apenas seus direitos. (...) provoca transtornos constantemente com suas reclamações e caprichos. (...) não obstante, mostra-se completamente indiferente para coisas importantes, como as relações com seus familiares próximos. Não gosta dos filhos, é incapaz de comportarse. (...) não se notou qualquer pista de sintomas paranóides ou catatônicos.

9. E. Bleuler. Dementia Praecox oder Gruppe der Schizophrenien. Leipzig und Wien, Franz Deuticke, 1911.

10. Idem. 


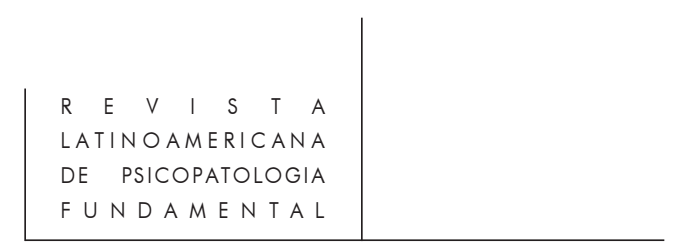

Em 1921, Rorschach ${ }^{11}$ reafirmará, como seu mestre Bleuler, a existência de forma latente de esquizofrenia. Zilborg ${ }^{12}$, por sua vez, em 1941 criou o termo esquizofrenia ambulatorial, para salientar a existência de formas frustas, quase não identificáveis de esquizofrenia, que em vez de ser encontradiça nos hospitais psiquiátricos, tendia a freqüentar os hospitais gerais e ambulatórios.

É entretanto, Stern ${ }^{13}$, em 1938, o primeiro autor a utilizar o termo borderline, num texto intitulado "Terapia e investigação psicanalítica do grupo das neuroses borderline". Stern descreveu pacientes que despertavam fortes reações contratransferenciais em seus terapeutas, pacientes que tendiam a regredir intensamente na falta de uma estrutura ambiental mais organizada, situação que eles, paradoxalmente, buscavam evitar.

Deutsch ${ }^{14}$, referindo-se a esses estados intermediários entre a psicose e a neurose, descreve, em 1942, a "personalidade como se". Segundo a psicanalista, essas pessoas apresentariam uma personalidade que, por trás de uma tênue adequação nos relacionamentos sociais do dia-a-dia, apresentavam um grave distúrbio nos relacionamentos interpessoais mais significativos.

Em 1949, Hoch e Polatin ${ }^{15}$ introduziram na nomenclatura o termo esquizofrenia pseudoneurótica, definido como uma condição psicopatológica específica, caracterizada pela combinação de "pan-neurose", "pan-ansiedade" e transtornos pervasivos da esfera sexual, sintomas esses associados às formas brandas de sintomas propriamente esquizofrênicos.

Nesse período, houve certa hesitação na nomeação dos quadros tidos como intermediários entre neurose e psicose. Vários termos foram sugeridos, tais como: pré-esquizofrenia, caráter esquizofrênico, esquizofrenia abortiva, esquizofrenia pseudopsicopática, caráter psicótico, esquizofrenia subclínica, síndrome borderland e esquizofrenia oculta.

É porém apenas em 1953, através do trabalho de Robert Knight ${ }^{16}$ "Estados Borderline", que o termo borderline começa a se firmar e a ganhar mais espaço na literatura psiquiátrica e psicanalítica. Knight utilizava o termo borderline para pacientes, geralmente encontrados no contexto da internação psiquiátrica, que não poderiam ser classificados nem como psicóticos, nem como neuróticos.

11. Cf. E. Brenas \& F. Ladame. "Les adolescents borderline: diagnose et traitement". Psychiatrie de l'enfant, 28 (2): 485-512, 1985.

12. Idem.

13. Cf. G.E. Vaillant \& J.C. Perry. "Personality disorders", in Kaplan, H.I. \& Sadock, B.J. Comprehensive Texbook of Psychiatry IV. Baltimore, William \& Wilkins, 1985.

14. Idem.

15. Idem.

16. Idem. 


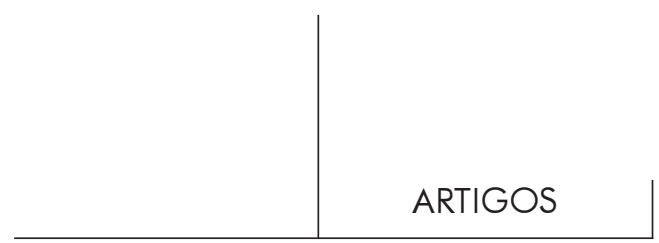

Não obstante, após o influente artigo de Knight, que trouxe crescente popularidade ao termo borderline, continuou a haver uma boa dose de desacordo em relação à sua definição precisa. Desde sua criação surgiram dúvidas, por exemplo, de se considerar o borderline ou como um tipo de paciente ou como um estado transitório, como uma organização de personalidade ou como uma forma grave de neurose, como um tipo atenuado de psicose ou como uma entidade nosológica de direito, ou, finalmente, tratar-se-ia apenas de uma rubrica para quadros clínicos que os clínicos e psicanalistas não conseguiam confortavelmente enquadrar nas categorias diagnósticas clássicas, já bem conhecidas. A tabela 1 resume a evolução histórica terminológica relativa ao transtorno borderline.

\begin{tabular}{|l|l|}
\hline \multicolumn{1}{|c|}{ Autor / ano } & \multicolumn{1}{|c|}{ Denominação } \\
\hline Kahlbaum, 1890 & Heboidofrenia \\
\hline Bleuler, 1911 & Esquizofrenia latente \\
\hline Rorschach, 1921 & Esquizofrenia latente \\
\hline Stern, 1938 & Neuroses borderline \\
\hline Zilborg, 1941 & Esquizofrenia ambulatorial \\
\hline Deutsch, 1942 & Personalidade "como se" \\
\hline Hoch \& Polatin, 1949 & Esquizofrenia pseudoneurótica \\
\hline Knight, 1953 & Estados borderline \\
\hline CID-9, 1976 & Esquizofrenia latente ou borderline \\
\hline DSM-III, 1980 & Transtorno de personalidade borderline \\
\hline CID-10, 1992 & $\begin{array}{l}\text { Transtorno de personalidade emocionante instável, } \\
\text { tipo borderline }\end{array}$ \\
\hline DSM-IV, 1994 & \begin{tabular}{l} 
Transtorno de personalidade borderline \\
\hline
\end{tabular} \\
\hline
\end{tabular}

Tabela 1: Evolução histórica do conceito borderline 


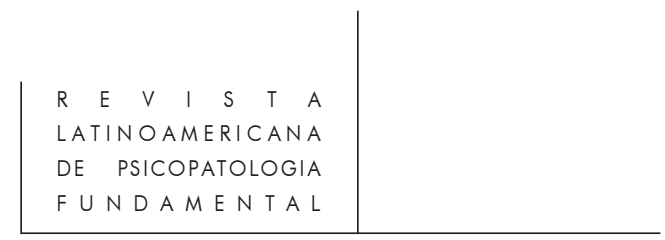

\section{As transformações do conceito de borderline: da CID-9 para o DSM-III}

É importante salientar que até os sintomas diagnósticos do DSM-II (APA, 1968) e da CID-9 (OMS, 1976) ${ }^{17}$ o termo borderline esteve situado dentro do espectro das psicoses, particularmente associado à esquizofrenia. No DSM-II e na CID-9 o termo borderline é praticamente sinônimo de esquizofrenia latente (CID 295.5).

$\mathrm{Na}$ sinonímia de esquizofrenia latente temos: esquizofrenia marginal, limítrofe ou borderline, esquizofrenia pré-psicótica, pseudopsicótica ou reação esquizofrênica latente. Explicitamente, a CID-9 descreve da seguinte forma o que classifica como esquizofrenia borderline ${ }^{18}$ :

Não foi possível formular uma descrição completamente aceitável deste quadro mental. Não se recomenda esta categoria para uso generalizado, entretanto, apresentase para aqueles que acreditam ser útil; trata-se de um quadro mental que se manifesta por meio de um comportamento excêntrico ou inconseqüente e de anomalias que dão a impressão de esquizofrenia, apesar de não haver manifestação, presente ou passada, de anomalias esquizofrênicas definidas e características. Os termos de inclusão indicam que este é o melhor lugar para classificar outras variedades de esquizofrenias mal definidas.

Assim, é de se notar o compromisso dessa definição com a tradição diagnóstica referente a estados esquizofrênicos frustos, "esquizofrenias sem sintomas esquizofrênicos clássicos", tradição que remonta claramente a Kahlbaum e Bleuler. Por outro lado, fica também evidente que a CID-9 reconhece o caráter precário desta categoria, sugerindo que aí encontre acolhida todas aquelas formas de esquizofrenia mal definidas e obscuras.

É apenas com o advento de novo sistema diagnóstico de 1980, o DSM-III ${ }^{19}$, que a situação nosotáxica e nosológica do quadro borderline vai se definir de modo radicalmente novo.

O DSM-III ${ }^{20}$ introduz mudanças substanciais na concepção dos sistemas diagnósticos modernos. Propõe-se a ser um sistema de classificação com cinco eixos de avaliação:

1. Transtorno psiquiátrico principal;

2. Transtorno de personalidade ou, para crianças e adolescentes, transtornos do desenvolvimento;

17. CID-9 (OMS). Nona Revisão da classificação Internacional de Doenças. Organização mundial para a Saúde, Genebra, 1976.

18. Idem

19. DSM-III (APA) (1980). Op. cit.

20. Idem. 


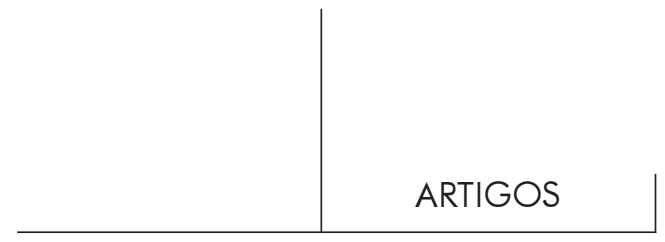

3. Doenças físicas;

4. Estressores psicossociais;

5. Nível de adaptação social antes da eclusão do distúrbio.

Além dessa concepção penta-axial, o DSM-III foi concebido para ser um sistema diagnóstico ateórico no que se refere à etiologia dos diversos distúrbios mentais. Tal princípio facilitaria, segundo seus defensores, uma maior concordância (reability) entre clínicos e pesquisadores no uso das categorias diagnósticas do sistema. Finalmente, o DSM-III exige, para a realização de determinado diagnóstico, critérios específicos e explícitos de inclusão e exclusão.

É inegável que o DSM-III norte-americano significou um grande avanço no sentido de tornar as categorias diagnósticas e seu uso reprodutíveis, apostando na estabilidade e clareza de um modo de comunicação que hipervaloriza os comportamentos explícitos e os sintomas inequívocos. Busca, sobretudo, afastarse das possíveis ambigüidades das expressões clínicas subjetivas, daquilo que o clínico percebe como implícito e de difícil definição no quadro clínico dos doentes. Rejeita, desta forma, a intuição do entrevistador como elemento diagnóstico fundamental, o feeling, o praecox Gefuehl dos psicopatólogos clássicos. Assim, o DSM-III é, de certa forma, o turning point de uma psiquiatria baseada em grandes mestres e escolas para uma psiquiatria sustentada em métodos epidemiológicos e no consenso de grupos de trabalho. O triunfo da psiquiatria pragmática sobre a doutrinária, da empiricista sobre a teorizante.

Por outro lado, deve-se ressaltar que apesar das qualidades desse tipo de sistema diagnóstico, alguns autores apontaram críticas que devem aqui ser mencionadas. Por exemplo, Lefèvre e cols. ${ }^{21}$ salientaram que apenas explicitamente o DSM-III é ateórico; implicitamente, porém, trata-se de um modelo comportamental, objetivista e naturalista da doença mental, que se fundamenta quase que exclusivamente sobre os comportamentos manifestos e as ocorrências externas, em detrimento do mundo interno das vivências mais íntimas e subjetivas do sujeito acometido pelo transtorno mental. Seria também ingênuo, do ponto de vista epistemológico, postular que é possível a observação e classificação inequívoca de fenômenos humanos, de forma neutra e ateórica, livre de qualquer a piori teórico ou conceitual. Os fenômenos psicopatológicos são de fato, na maioria das vezes, prenhes de significações simbólicas cambiantes, e freqüentemente "contaminados" por uma subjetividade tal, que torna a sua objetivação, operacionalização, universalização e quantificação empírica, no mínimo, problemática.

21. M. Lefèvre; S. Lebovici \& P. Jeammet. "L'amplication de la nouvelle classification américane dite DSM-III a la psychiatrie de l'enfant e de l'adolescent". Psychiatrie de l'enfant, 26 (2): 1983. 


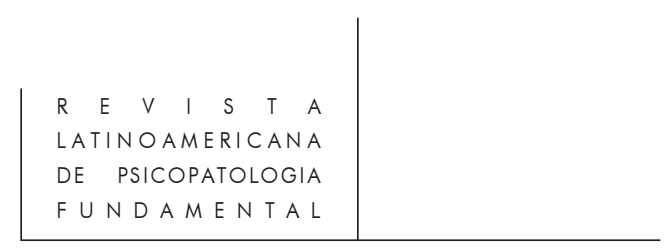

Tomadas essas restrições de cunho epistemológico, há um certo consenso de que o DSM-III representou, principalmente no campo da pesquisa epidemiológica e psicobiológica, um avanço considerável em relação à CID-9 e ao DSM-II. A confiabilidade dos diagnósticos melhorou de forma evidente, além da precisão e clareza na comunicação entre clínicos e pesquisadores de diversos países.

O que é particularmente relevante para o assunto aqui tratado, é que no DSM-III de 1980, o quadro borderline sai definitivamente do espectro da esquizofrenia para migrar, numa viagem aparentemente sem retorno, para o capítulo dos distúrbios da personalidade. A esquizofrenia latente ou borderline irá, de fato, dar origem a dois tipos de distúrbios de personalidade: o distúrbio esquizotípico de personalidade e o distúrbio borderline de personalidade.

\begin{tabular}{|l|l|}
\hline Espectro da esquizofrenia & Espectro do distúrbio de personalidade \\
\hline Esquizofrenia latente ou borderline & $\begin{array}{l}\text { Distúrbio de personalidade esquizotípico } \\
\text { Distúrbio de personalidade borderline }\end{array}$ \\
\hline
\end{tabular}

\section{Evolução nosotáxica da esquizofrenia latente}

Tal mudança não ocorreu de modo gratuito ou aleatório. Para tal contribuiu de modo decisivo o artigo de Spitzer, Endicott e Gibbon ${ }^{22}$, de 1979, intitulado "Crossing the border into borderline personality and borderline schizofrenia". Os autores partiram da constatação de que o termo borderline era utilizado pelos psiquiatras em duas linhas diferentes. A primeira referia-se a uma constelação de pacientes, nos quais havia importantes traços de personalidade de instabilidade e vulnerabilidade à frustração, que eram significativos para a evolução e o tratamento. Exemplos desse uso do conceito são os trabalhos de Gunderson e cols. ${ }^{23}$ (1975) sobre "pacientes borderline" e os de Kernberg ${ }^{24}$, sobre a "organização de personalidade de borderline".

A segunda linha de trabalhos referia-se ao uso do termo para descrever certas características psicopatológicas que são usualmente estáveis ao longo do tempo, como comportamento bizarro, idéias deliróides, de auto-referência, às quais se

22. R. Spitzer; J. Endicott \& M. Gibbon. "Crossing the border into borderline personality and borderline schizophrenia”. Arch. Gen. Psychiatry, 36, 1979.

23. J.G. Gunderson; W.T. Carpenter \& J.S. Strauss. "Borderline and schizophrenic patients: a comparative study". Am. J. Psychiatry, 132 (12), 1975.

24. Cf. E. Brenas \& F. Ladame. Op. cit. 


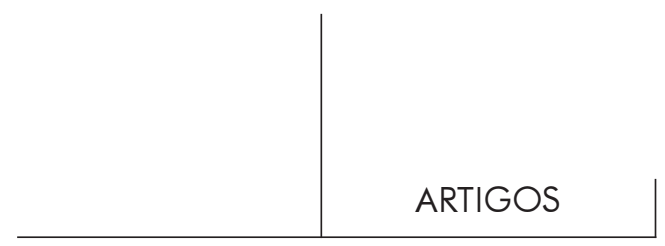

postulava serem geneticamente relacionadas ao espectro da esquizofrenia. Este uso foi utilizado por Wender e cols. ${ }^{25}$, em seus estudos sobre crianças adotadas e sua relação com a esquizofrenia.

O estudo do grupo de Spitzer $(1979)^{26}$ teve por objetivo definir operacionalmente o que seria mais adequado: o uso de termos e conceitos separados para cada uma dessas duas grandes linhas, ou se uma única categoria nosológica poderia abarcar as duas vertentes.

Após um detalhado estudo utilizando questionários específicos para testar estatisticamente a viabilidade ou não de discriminação dos dois grandes grupos, Spitzer e cols. (1979) ${ }^{27}$ concluíram pela hipótese de duas entidades diagnósticas. Assim, verificou-se que o conceito de borderline, usado até então, englobava de fato dois grupos de pacientes distintos e relativamente independentes entre si.

No primeiro grupo confirmou-se a existência de distúrbio de identidade, padrão de relações interpessoais instáveis e intensas, impulsividade e imprevisibilidade dos atos, raiva inapropriada e perda de controle sobre a raiva, atuações autodestrutivas, baixa performance escolar ou profissional para o nível de inteligência, instabilidade afetiva marcante, sentimentos crônicos de vazio e intolerância de estar só. Inicialmente denominaram tal grupo de "personalidade instável" e, posteriormente, de "personalidade borderline" 28 . Tal grupo teria talvez (e pesquisas posteriores reforçam tal hipótese) um certo "parentesco" genético, portanto, nosológico, com transtornos afetivos.

O segundo grupo seria o de pacientes nos quais se encontravam com maior frequiência: comunicação bizarra, idéias de referência, ideação paranóide, sensação de presença de força ou pessoa estranha, pensamento mágico, rapport inadequado com certa inadequação afetiva, hipersensibilidade à crítica e isolamento social. Tal grupo foi denominado de "personalidade esquizotípica", sendo então sugerido, baseado em algumas constatações, que havia uma relação genética e nosológica entre esse quadro e a esquizofrenia ${ }^{29}$.

Assim, o grupo de Spitzer ${ }^{30}$ conclui que o que era anteriormente denominado "esquizofrenia borderline ou latente" deveria receber a rubrica de "transtorno esquizotípico de personalidade", e aquelas pessoas denominadas por vários autores como "pacientes borderline", deveriam ser classificadas como portadores de um

25. Cf. R. Spitzer; J. Endicott \& M. Gibbon. Op. cit.

26. Idem.

27. Idem.

28.M. Steiner; P.S. Links \& M. Korzekwa. "Biological markers in borderline personality disorders: an overview". Can. J. Psychiatry, 33: 350-354, 1988.

29.Idem.

30.R. Spitzer; J. Endicott \& M. Gibbon. Op. cit. 


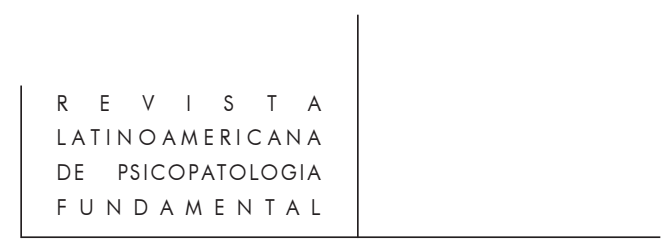

distúrbio grave de personalidade, o então chamado "transtorno de personalidade tipo borderline". Desta forma, estes dois novos grupos, mais bem distintos e delimitados, foram incorporados ao DSM-III permanecendo, daí em diante, nas novas edições do DSM-III (o DSM-III-R e o DSM-IV ${ }^{31}$ ).

As duas grandes categorias foram também, em 1992, incorporadas à nova classificação de transtornos mentais da OMS, a CID-10 $10^{32}$. Aqui, entretanto, a CID-10 opta por um ordenamento nosotáxico um tanto distinto do DSM-IV. A CID-10 mantém o transtorno esquizotípico no capítulo do espectro esquizofrênico, enfatizando assim o seu caráter de transtorno aparentado com a esquizofrenia clássica. Apenas o transtorno de personalidade tipo borderline fica definitivamente no capítulo dos transtornos de personalidade emocionalmente instável. Nessa categoria a CID-10 agrupa dois subtipos: o tipo impulsivo e o tipo borderline. Nas páginas 61 e 62 vemos nas tabelas 2 e 3 as características e sintomas desses dois transtornos, originados da noção de esquizofrenia latente.

\section{O transtorno de personalidade tipo borderline: aspectos clínicos e psicopatológicos}

Epidemiologia

Os transtornos de personalidade, de modo geral, são considerados bastante freqüentes pela epidemiologia psiquiátrica. Segundo Gunderson \& Phillips $(1995)^{33}$, estima-se que aproximadamente 10 a $13 \%$ da população geral, quando investigada com instrumentos epidemiológicos, preenche os critérios diagnósticos para transtorno de personalidade. Casey $(1988)^{34}$, identificou uma prevalência de cerca de $4 \%$ para as formas mais graves de transtorno de personalidade (incluindo o transtorno borderline, a sociopatia, e outros transtornos de personalidade com intenso distúrbio nas relações interpessoais, hostilidade extrema, comportamentos auto e heterodestrutivos marcantes etc.). As taxas são mais altas nos estratos mais pobres da população e em comunidades marginalizadas, onde predomina a violência e a desagregação familiar.

31. DSM-IV (APA). Diagnostic and Statistical Manual of Mental Disorders, 4th ed.. American Psychiatric Association, Washington, DC,1994.

32. ICD-10 (WHO). The ICD-10 Classification of Mental and Behavioral Disorders: Diagnostic Criteria for Research. World Health Organization, Geneve, 1993.

33. J.G. Gunderson \& K.A. Phillips. "Personality disorder", in Kaplan, H.I. \& Sadock, B.J. Comprehensive Textbook of Psychiatry VI. Baltimore, Willians \& Wilkins, 1995.

34. P. Casey. "The epidemiology of personality disorder", in Personality Disorder: Diagnosis, Management and Care, P. Tyrer (ed.). Londres, Wright, 1988, pp. 74-81. 


\section{ARTIGOS}

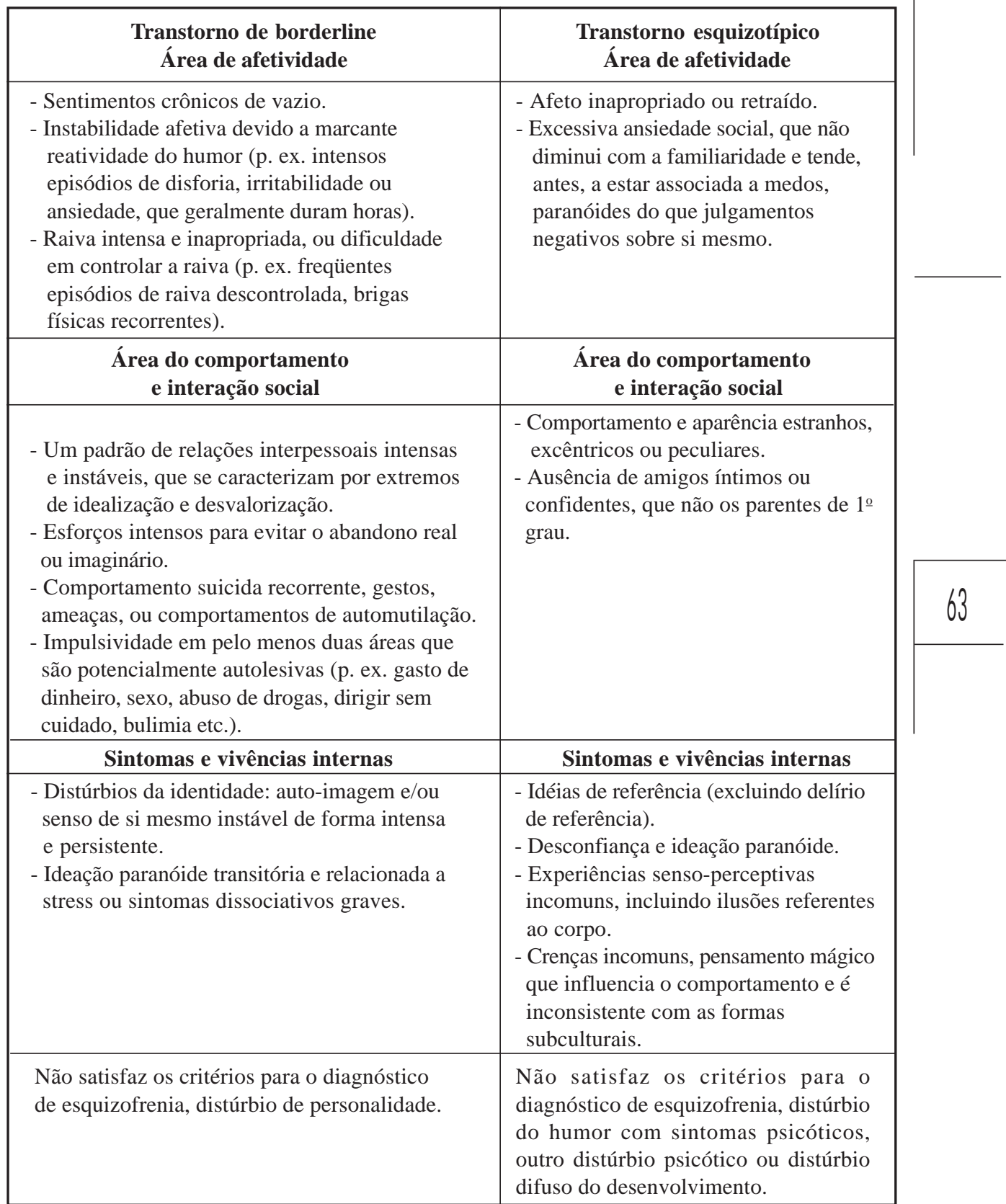

Tabela 2: Transtornos de personalidade borderline e esquizotípico, segundo o DSM-IV 


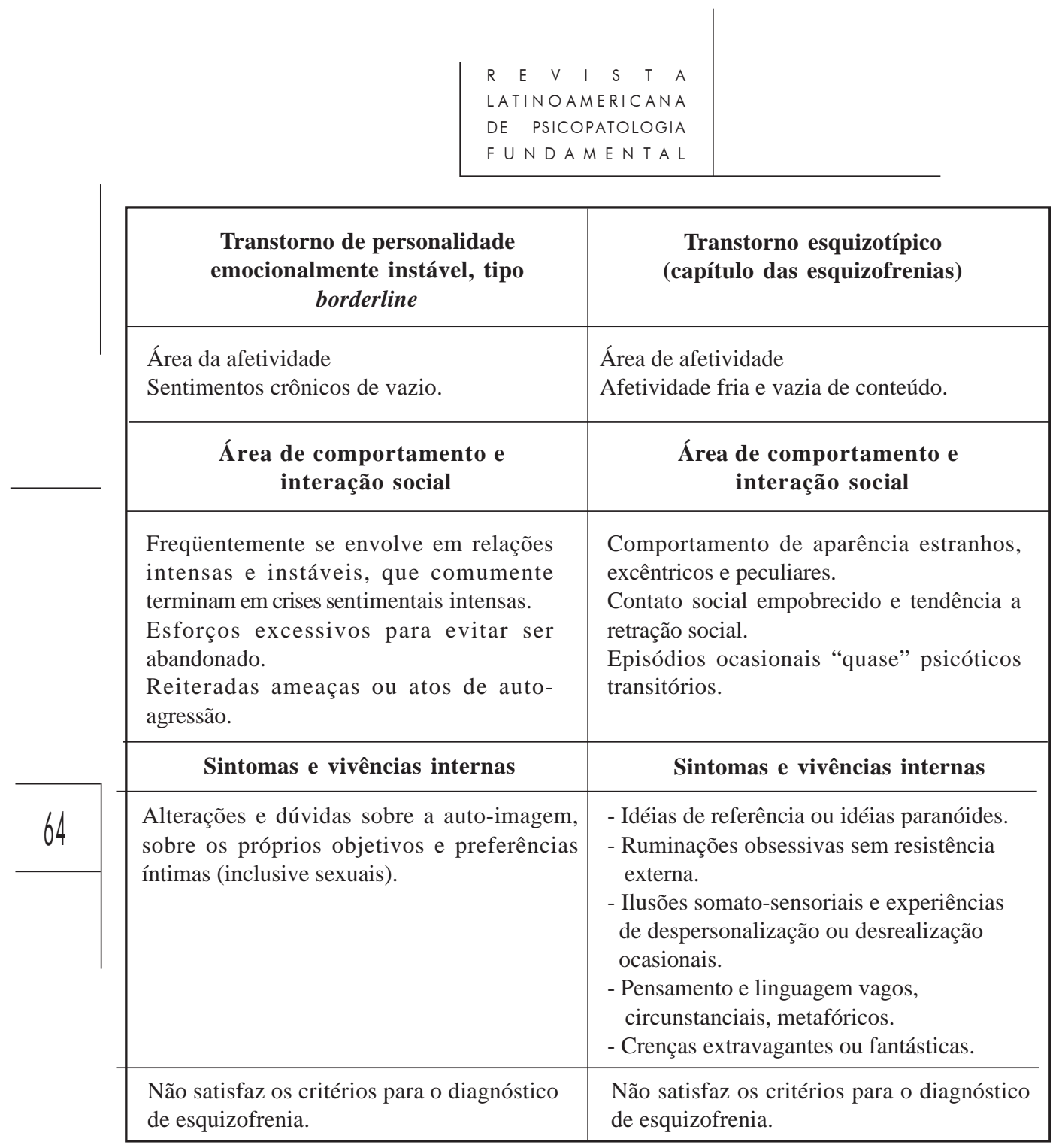

Tabela 3: Transtornos de personalidade de borderline e transtorno esquizotípico, segundo a CID-10 (critérios de pesquisa)

Nos grupos de pacientes psiquiátricos internados, estima-se que cerca de 15\% são casos cujo diagnóstico principal é um grave transtorno de personalidade, e cerca da metade de todos os pacientes internados apresentam algum transtorno de personalidade acompanhando o diagnóstico psiquiátrico principal, como comorbidade. Em grupos ambulatoriais, a prevalência de transtornos de personalidade fica geralmente na faixa de 30 a $50 \%$. Isto varia, entretanto, com o perfil da clínica estudada. Por exemplo, programas para dependentes de drogas revelam uma 


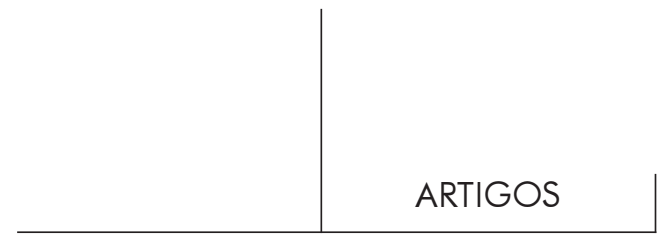

porcentagem bem mais alta que ambulatórios de psicoterapia. $\mathrm{O}$ curso do transtorno borderline, após a adolescência e período inicial da vida adulta, parece indicar certa estabilização nos relacionamentos interpessoais e no trabalho.

\section{Psicopatologia}

É interessante constatar, no tratado de psiquiatria de Eugen Bleuler ${ }^{35}$, atualizado por seu filho Manfred Bleuler - no qual ainda se utiliza o conceito de borderline como transição entre neurose e psicose - como a noção de esquizofrenia borderline vai tomando uma configuração mais próxima da atual noção de transtorno de personalidade borderline:

As perturbações fronteiriças (borderline) constituem-se primordialmente, nas descargas afetivas impulsivas, na irregularidade e contradição nas relações afetivas, na insegurança a respeito da própria identidade, nas disforias e, ocasionalmente, nas ideações deliróides, apesar de faltar a confusão esquizofrênica.

Já Vaillant \& Perry $(1985)^{36}$ salientam que o comportamento das pessoas com personalidade borderline é em boa medida imprevisível. Notam a natureza trágica de suas vidas, evidenciada nas repetidas ações autodestrutivas: "cortam os pulsos e praticam outras automutilações com o objetivo de atrair atenção, exprimir raiva ou entorpecer-se ante o afeto esmagador".

Devido a dependência e hostilidade intensas, as relações interpessoais são, geralmente, muito tumultuosas. A evolução do quadro clínico a longo prazo ainda é pouco conhecida, porém é intrigante que tal diagnóstico seja menos freqüente em adultos depois dos quarenta anos e em idosos. Talvez seja um indício de que o transtorno de personalidade borderline seja um distúrbio próprio de adolescentes e adultos jovens, e que no adulto maduro e no idoso esse perfil de personalidade tomaria "um outro rumo". Mais recentemente, Garnet e cols. (1994) ${ }^{37}$ têm conduzido estudos no sentido de elucidar a estabilidade da personalidade borderline, especificamente em adolescentes. Ainda resta certa controvérsia nesta área (Links e cols., 1993; Zaimes, 1995) $)^{38,39}$.

35. E. Bleuler \& M. Bleuler. Psiquiatria. Rio de Janeiro, Guanabara Koogan, 1985.

36. G.E. Vaillant \& J.C. Perry. Op. cit.

37. K.E. Garnet; K.N Levy; J.J.F. Mattanah; W.S. Edell \& T.H. McGlashan. "Borderline personality disorder in adolescents: ubiquitous or specific?” Am. J. Psychiatry, 151(9): 1380-1382, 1994.

38. P.S. Links; M.J.E. Mitton; M. Steiner. "Stability of borderline personality disorder". Can. J. Psychiatry, 38: 255-259, 1993.

39. J.M.S. Zaimes. “Adolescent borderline personality disorder”. Am. J. Psychiatry, 152: 1537-1538, 1995. 


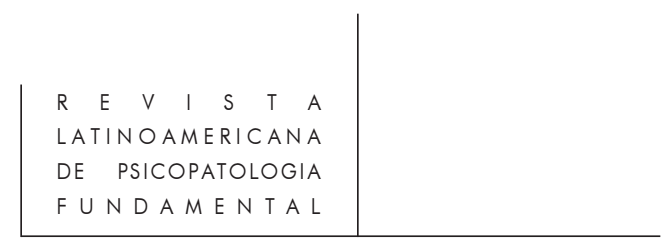

No diagnóstico diferencial a principal distinção a fazer é com a personalidade esquizotípica, sendo que outros quadros devem ser também afastados, tais como os transtornos de personalidade tipo histriônico, anti-social, esquizóide ou paranóide, os transtornos afetivos maiores e também a própria esquizofrenia, nas suas apresentações com poucos e discretos sintomas.

A fim de precisar o diagnóstico, deve-se ter em mente que o que é típico do transtorno de personalidade borderline são os seguintes fenômenos clínicos: sentimentos crônicos de vazio, impulsividade, automutilação, episódios psicóticos de curta duração, tentativas manipuladoras de suícidio e, freqüentemente, relações interpessoais muito conturbadas e insatisfatórias.

Deve-se salientar, também, que apesar de boa parte da literatura ter enfocado os sintomas psicóticos de curta duração, seus sintomas são, no mais das vezes, circunscritos, fugazes ou duvidosos.

A seguir será exposto um resumo dos aspectos clínicos do transtorno borderline, segundo a linha de alguns autores com enfoques semelhantes (Kroll, 1981; Pope, 1983; Macaskilli, 1985; Southwick e cols., 1995) ${ }^{40,41}$.

\section{Sintomas mais característicos:}

afetividade, comportamentos e sintomas psicóticos ${ }^{42,43}$

\section{Afetividade}

- Raiva e ira: são muito comuns, dirigidas a uma variedade de pessoas e/ou objetos. Elas parecem, muitas vezes, constituir os principais ou únicos afetos que o paciente experimenta;

- Depressão e ansiedade: geralmente não se trata de uma depressão do tipo culposa, mas muito mais relacionada a sentimentos de profunda solidão e rejeição. Há uma vivência predominante de sentir-se fútil, só, isolado e rejeitado pelas pessoas de seu convívio ${ }^{44}$. Neste sentido, a depressão do indivíduo borderline se assemelha,

40. J. Kroll. "Borderline personality disorder: Construct validity of the concept". Arch. Gen. Psychiastry, 38, 1981.

41. S.M. Southwick; R. Yehuda \& E.L. Giller. "Psychological dimensions of depression in borderline personality disorder". Am. J. Psychiatry, 152(5): 789-781, 1995.

42. J.H. Nelson. "Comparison of three system for diagnosing borderline personality disorder". Am. J. Psychiatry, 142(7), 1985.

43. E.R. Shapiro. "The psychodynamics and developmental psychology of the borderline patient: a review of the literature". Am. J. Psychiatry, 135(11), 1978.

44. J.C. Perry. "Depression in borderline personality disorder: Life-time prevalence at interview and longitudinal course of symptoms". Am. J. Psychiatry, 142(1), 1985. 


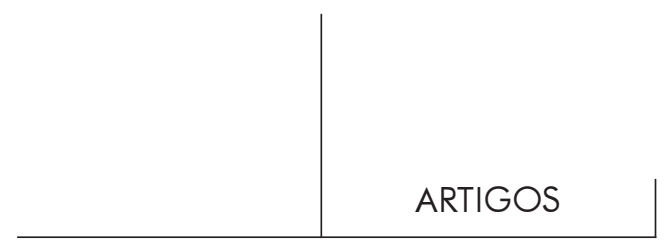

em parte, à vivência da depressão atípica. Alguns autores ressaltam a freqüência de sintomas ansiosos entre os pacientes borderline, geralmente associada a anedonia.

Em resumo, a experiência afetiva do paciente borderline é caracterizada pela proeminência da raiva e da depressão (solidão-vazio), e algum grau de ansiedade e anedonia. Tais pacientes tendem, de fato, a experimentar afetos intensos, intempestivos e instáveis, entretanto raramente suas vivências afetivas inclui a experiência do prazer.

Recentemente, Southwick e cols. $(1995)^{45}$ procuraram testar a hipótese de que o transtorno borderline seria mais freqüentemente acompanhado de depressão tipo anaclítica do que de depressão auto-acusatória. A depressão anaclítica ocorreria em pessoas dependentes, com tendência a sentimentos de desamparo, fraqueza, medo de abandono e uma forte necessidade de se sentirem protegidas e amadas. Já na depressão do tipo auto-acusatória ou introjetiva haveria o predomínio de autocrítica, sensação de menos valia, sentimentos de culpa e inferioridade. Os autores não puderam confirmar empiricamente sua hipótese, mas verificaram que os pacientes borderline tendem a experimentar na depressão uma autocrítica muito intensa, que tem sido negligenciada pela maior parte dos pesquisadores anteriores.

A característica mais freqüente e saliente do comportamento dos pacientes borderline é o caráter impulsivo e autodestrutivo de seus atos ${ }^{46}$. O termo autodestrutivo é usado para indicar um largo espectro de comportamentos que resultam ser autodestrutivos, embora seu objetivo inicial, às vezes, não seja esse. Exemplos incluem a promiscuidade sexual e perversões na busca de afeto, a automutilação com o objetivo de manipulação dos outros, de chamar a atenção e envolvimento com drogas, na tentativa de obter uma entidade provisória (de "viciado") ou, simplesmente, como forma de fuga e evitação de sentimentos de abandono.

É comum, também, uma certa confusão básica e dificuldade em relação à identidade sexual.

Sintomas psicóticos

Há certa concordância de que a síndrome borderline seja um distúrbio estável de personalidade. Entretanto, alguns desses pacientes podem desenvolver sintomas

45. S.M. Southwick; R. Yehuda \& E.L. Giller. Op. cit.1.

46. F. Masterson. Op. cit. 


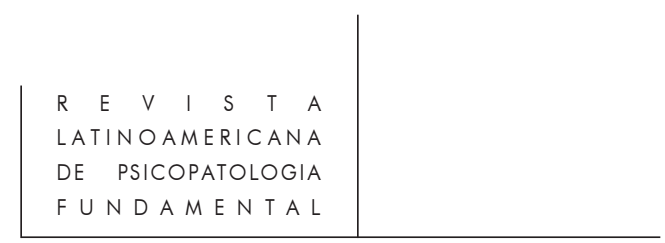

psicóticos transitórios ${ }^{47}$. Quando esses quadros psicóticos ocorrem, eles tendem a se apresentar com as seguintes características:

- relacionados a situação de estresse:

- são rapidamente reversíveis e transitórios;

- os sintomas psicóticos tendem a ser não-sitematizados;

- as experiências psicóticas tendem a ser ego-ditônicas.

De modo geral, há ausência de idéias delirantes e alucinações claras e estáveis. Há, antes, a vivência de perplexidade intensa, confusão e ideação paranóide pouco estruturada. Esses pacientes tendem a apresentar grande vulnerabilidade a estresses farmacológicos, como aqueles produzidos pela maconha, LSD, mescalina ou outros alucinógenos.

\section{Aspectos intrapsíquicos $\mathbf{4 8}^{\mathbf{8 9}}$}

A dimensão intrapsíquica pode ser avaliada em pesquisas através do uso de testes psicológicos projetivos. Nos testes não-projetivos, nos quais se avalia a performance cognitiva como o WAIS, os pacientes borderline tendem a demonstrar uma performance normal ${ }^{50}$. Entretanto, um estudo usando uma bateria mais sofisticada, incluindo uma avaliação de funções pré-frontais que cobrem dimensões como a flexibilidade cognitiva, as funções executivas e de controle, revelou uma performance prejudicada no grupo de pacientes borderline.

Em testes projetivos, como o teste de Rorschach, os pacientes borderline tendem a apresentar conexão ilógica de percepções não relacionadas, elaboração excessiva do sentido afetivo das percepções, produções e associações mentais que, embora sejam criativas, são muito desprazerosas e circunstancias.

\section{Funções egóicas ${ }^{51,52}$}

É no campo das relações interpessoais que se revelam as principais alterações das funções egóicas dos pacientes borderline ${ }^{53}$. Aqui seria a área mais propícia para

47. J.H. Nelson. Op. cit.

48. Idem.

49. E.R. Shapiro. Op. cit.

50. Idem.

51. J.H. Nelson. Op. cit.

52. E.R.Shapiro. Op. cit.

53. Cf. E. Brenas \& F. Ladame. Op. cit. 


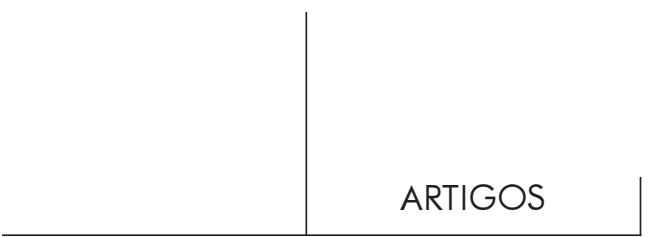

caracterizar com certa especificidade os indivíduos borderline, em relação a pacientes com outros transtornos de personalidade ${ }^{54}$.

Freqüentemente assinala-se que as relações interpessoais dos borderline são muito transitórias e superficiais. A qualidade dessa transitoriedade e superficialidade é melhor revelada pela hipótese original de Helen Deutsch, da "personalidade como se" ${ }^{55}$.

A característica essencial da "personalidade como se", segundo Deutsch, é que externamente tais pacientes conduzem sua vida relacional como se possuíssem uma capacidade emocional completa e sensível. Com facilidade, a ausência de uma resposta emocional real, esperada pelo paciente, ou mesmo frustrações insignificantes na esfera interpessoal, conduz à rápida dissolução das relações afetivas significativas. A grande distância entre relações superficiais adequadas e relações internas e íntimas inadequadas e muito insatisfatórias foi utilizada para caracterizar os pacientes borderline de forma mais expressiva.

Observa-se, também, que tais pacientes estão permanentemente prontos para formar relações pessoais intensas, invasivas e "aderentes" (Brenas \& Ladame, $1985)^{56}$. No dia-a-dia as relações interpessoais podem se apresentar estáveis, mas tendem a ser sempre superficiais. Quando se observam os borderlines em duas relações mais significativas e íntimas é que se nota como elas são instáveis, intensas, tendendo à manipulação e a dependência. São as relações interpessoais intensas que diferenciam claramente os borderlines dos indivíduos com personalidades esquizóde. Já a ausência de sintomas psicóticos estáveis (como ideação bizarra ou paranóide, percepção alterada, comportamento e atitudes extravagantes), os diferencia das personalidades esquizotípica e dos esquizofrênicos.

Finalmente, através das relações entre pacientes borderline e psicoterapeutas, revelam-se traços importantes do funcionamento mental desses pacientes. Tendem também a formar relações intensas e dependentes que, entretanto, logo descambam para relações manipulativas e desvalorizadoras (Masterson, 1972; Shapiro, 1978) 57, $58 .^{\text {. }}$ Vários autores ${ }^{59,60}$ têm ressaltado a importância do setting no qual esses pacientes são observados. Tais pacientes teriam grande probabilidade de se desestruturarem em settings pouco protetores, pouco estruturados.

54. E.R. Shapiro. Op. cit.

55. Cf. E. Brenas \& F. Ladame. Op. cit.

56. Idem.

57. F. Masterson. Op. cit.

58. E.R. Shapiro. Op. cit.

59.E. Brenas \& F. Ladame. Op. cit.

60. E.R. Shapiro. Op. cit. 


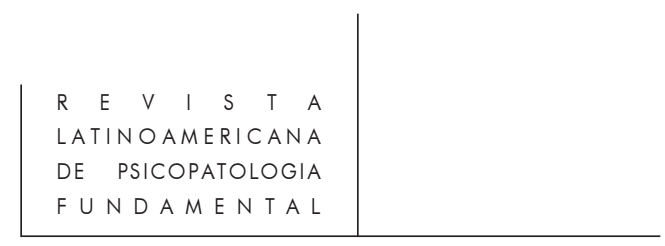

A psicoterapia intensiva, em settings estruturados, tem sido preconizada por muitos autores ${ }^{61,62}$. Paralelamente aos tratamentos psicoterápicos, têm-se pesquisado $^{63}$ uma série de psicofármacos (antipsicóticos tradicionais e atípicos, antidepressivos, sais de lítio, carbamazepina etc.) no sentido de se obter uma melhora tanto sintomática como de padrões de conduta. De modo geral, o transtorno borderline é considerado um transtorno de personalidade muito grave ${ }^{64}$, situado provavelmente no final do espectro de gravidade dos transtornos de personalidade ${ }^{65}$.

A autonomia nosológica e utilidade clínica do construto "transtorno borderline" ainda não estão suficientemente consolidados. Há inúmeros grupos de pesquisadores utilizando métodos da psiquiatria biológica, da epidemiologia e da psicanálise, visando precisar e aprofundar este construto. A investigação psicopatológica aprofundada, tanto de correntes fenomenológicas-descritivas, como de orientações psicanalíticas, certamente poderá contribuir para o esclarecimento da questão; a que veio, afinal, esta entidade nosológica enigmática na história recente da psicopatologia? $?^{66,67,68,69}$

61. D.F. Dawson. "Treatment of the borderline patient, relationship management". Can. J. Psychiatry, 33: 370-374, 1988.

62. A.B. Eppel. "Inpatient day hospital treatment of the borderline: an integrated approach". Can. J. Psychiatry, 33: 360-363,1988.

63. P.S. Links \& M. Steiner. "Psychopharmacologic management of patients with borderline personality disorder". Can. J. Psychiatry, 33: 355-359, 1988.

64. P.S. Links; M. Steiner; D.R. Offord \& A. Eppel. "Characteristics of borderline personality disorder: a Canadian study". Can. J. Psychiatry, 33: 336-340, 1988.

65. Cf. G.E. Vaillant \& J.C. Perry. Op. cit.

66. Cf. M. Steiner; P.S. Links \& M. Korzekwa.

67. Cf. K.E. Garnet; K.N Levy; J.J.F. Mattanah; W.S. Edell \& T.H. McGlashan.

68. Cf. P.S. Links \& M. Steiner. Op. cit.

69. Cf. M. Baron. "Familial transmission of schizotypal and borderline personality disorders". Am. J. Psychiatry, 142(8), 1985. 


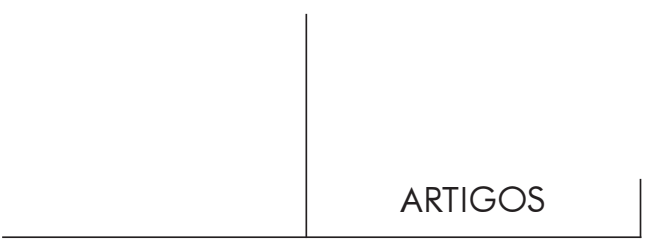

Resumos

En la presente investigación es echa una revisión histórica y psicopatologica del disturbio borderline. El autor sugiere que la categoria clínica introducida por Kahlbaum, en 1890, "heiboidophenie", puede ser considerada como un protoconcepto en el origen del concepto de transtorno borderline. A fines de los años 70 y 80, bajo la influência del DMS-III, la esquizofrenia latente o borderline "migrava" hacia dos subtipos de transtorno de personalidad: borderline y esquizotípico. En este trabajo son presentados, con especial ênfasis a los sintomas afetivos e las alteraciones en el plan de la interación social.

Palabras-llave: Transtorno de personalidad borderline, esquizofrenía latente, historia de la psicopatología.

Dans ce travail on fait la révision historique et psychopathologique de le trouble borderline. L'auteur suggére qui la catégorie clinique introduite pou Kahlbaum, en 1890, "heiboidophenie", peut être considerée comme un protoconcept dans l'origine du concept de bouleversement borderline. À la fin des années 70 et 80, sous l'influence de le DMS-III, la schizophrénie latent ou borderline se muait en deux sous-types de bouleversement de personnalité: borderline et schizotypique. Quelques-uns aspects psychopathologiques de le Bouleversement de la Personnalité borderline sont revues, avec spécial emphase sur les symptômes affectifs et les changements dans le plan de les attaches interpersonnelles.

Mots-clef: Bouleversement de la personnalité borderline, schizophrénie latent, histoire de la psychopathologie.

This paper reviews the historical origins and psychopathological dimensions of the borderline disorder. The author suggests that Kahlbaum's clinical category "Heiboidophrenie", introduced in 1890, is an eligible candidate as a protoconcept in the history of latent schizophrenia and borderline disorders. In the late 1970's and 1980 's, under the influence of DSM-III, latent or borderline schizophrenia split into two personality disorders: schizotype and borderline. Some psycopathological aspects of borderline personality disorder are presented, concerning specially affective symptoms ad social interaction disruption.

Key words: Borderline disorder, latent schizophrenia, history of psychopathology. 